\title{
Tortile Yang-Baxter operators for crossed group-categories
}

\author{
Takeo FuKushI \\ (Received September 4, 2000; Revised December 13, 2000)
}

\begin{abstract}
The notion of a tortile Yang-Baxter operator in a crossed group-category is introduced. It is shown that a tortile Yang-Baxter operator on an object $X$ induces a unique braiding and a twist on the free crossed group-category generated by the objects $X$ and $X^{*}$.
\end{abstract}

Key words: tortile Yang-Baxter operator, crossed group-category.

\section{Introduction}

The category of tangles in 3 dimension has a beautiful algebraic characterization in terms of a universal property. This was initially developed by Yetter [10], Turaev [8], Freyd-Yetter [1] and Joyal-Street [3], and has culminated in the work of Shum [7] asserting that the category of framed tangles $\mathcal{F} \mathcal{T}$ is monoidally equivalent to the tortile category freely generated by a single object. Joyal and Street [2] gave another purely algebraic interpretation of this category as the free tensor category containing an object equipped with a tortile Yang-Baxter operator.

Recently, Turaev [9] introduced the notion of a modular crossed groupcategory, and used it to develop 3-dimensional homotopy quantum field theory (HQFT). He started with defining the notion of a tortile (ribbon) crossed $\pi$-category for a group $\pi$, and showed that modular crossed $\pi$-categories induce invariants of 3 -dimensional $\pi$-manifolds.

The aim of this paper is to give the Joyal and Street's interpretation for a crossed group-category. To do this, we define a balanced Yang-Baxter operator and a tortile Yang-Baxter operator in a crossed group-category. Then we prove that the free crossed group-category $\mathcal{F}$ generated by a single object equipped with a tortile Yang-Baxter operator admits a unique braiding and a twist. Although our construction owes much to the paper [2], several new aspects appear. First, it turns out that one should define a twist 
before a Yang-Baxter operator. This statement means that in a general crossed group-category, it is not possible to define a Yang-Baxter operator without a twist. Thus one can define only balanced Yeng-Baxter operators in a crossed group-category. Second, we use the fact that the category $\mathcal{F}$ admits a connectivity structure, which we feel non-trivial. In general, for an object $U$ in a crossed $\pi$-category $\mathcal{C}$, the centralizer $\mathcal{L}_{\mathcal{C}}(U)$ does not admit a crossed $\pi$-category structure. However, if a crossed $\pi$-category $\mathcal{C}$ is connected, then the category $\mathcal{L}_{\mathcal{C}}(U)$ admits a crossed $\pi$-category structure, so that we can aplly this procedure to $\mathcal{F}$. Third, since we have to consider a Yang-Baxter operator with a twist, various identities which were simple in [2] become much more complicated. To overcome this difficulty, we use a diagrammatic notion. Then, we can check that each equality between diagrams corresponds to a certain equality between morphisms in $\mathcal{F}$. As a result, we see that the constructions above are all well done and the theorem holds.

\section{Preliminaries}

Definition 1 Let $\pi$ be a group and let $\mathcal{C}$ be a strict monoidal category with a unit object $I$. Then the category $\mathcal{C}$ is called a $\pi$-category if it satisfies the following conditions:

(a) there are full subcategories $\mathcal{C}_{\alpha}(\alpha \in \pi)$ of $\mathcal{C}$ such that each object of $\mathcal{C}$ belongs to $\mathcal{C}_{\alpha}$ for a unique $\alpha \in \pi$;

(b) if $U \in \mathcal{C}_{\alpha}$ and $V \in \mathcal{C}_{\beta}$ with $\alpha \neq \beta$ then there is not any morphism from $U$ to $V$;

(c) $I \in \mathcal{C}_{1}$, and if $U \in \mathcal{C}_{\alpha}$ and $V \in \mathcal{C}_{\beta}$ then $U \otimes V \in \mathcal{C}_{\alpha \beta}$.

In [9] a $K$-additivity and a left duality are assumed in the monoidal category $\mathcal{C}$. In this paper, we do not assume those structures in $\mathcal{C}$.

Definition 2 In the setting above, an automorphism of $\mathcal{C}$ is defined as a functor $\varphi: \mathcal{C} \rightarrow \mathcal{C}$ wich preserves the tensor product and the unit object. Thus,

$$
\varphi(I)=I, \quad \varphi(U \otimes V)=\varphi(U) \otimes \varphi(V), \quad \varphi(f \otimes g)=\varphi(f) \otimes \varphi(g),
$$

for any objects $U, V$ and any morphisms $f, g$ in $\mathcal{C}$. We denote by $\operatorname{Aut}(\mathcal{C})$ the group of automorphisms of $\mathcal{C}$. A crossed $\pi$-category is a $\pi$-category $\mathcal{C}$ endowed with a group homomorphism $\varphi: \pi \rightarrow \operatorname{Aut}(\mathcal{C})$ such that for all 
$\alpha, \beta \in \pi$ the functor $\varphi_{\alpha}=\varphi(\alpha): \mathcal{C} \rightarrow \mathcal{C}$ maps $\mathcal{C}_{\beta}$ to $\mathcal{C}_{\alpha \beta \alpha^{-1}}$. For objects $U \in \mathcal{C}_{\alpha}, V \in \mathcal{C}_{\beta}$, set ${ }^{U} V=\varphi_{\alpha}(V)$.

For crossed $\pi$-categories $\mathcal{C}, \mathcal{C}^{\prime}$, a tensor functor $\mathcal{C} \rightarrow \mathcal{C}^{\prime}$ is called a crossed $\pi$-functor if it preserves the action of $\pi$.

Definition 3 Let $\mathcal{C}$ be a crossed $\pi$-category. A braiding in $\mathcal{C}$ is a system of invertible morphisms $c_{U, V}: U \otimes V \rightarrow{ }^{U} V \otimes U$ satisfying the following conditions:

(a) for any morphisms $f: U \rightarrow U^{\prime}$ and $g: V \rightarrow V^{\prime}$ such that $U, U^{\prime}$ lie in the same component of $\mathcal{C}$, we have

$$
c_{U^{\prime}, V^{\prime}}(f \otimes g)=\left({ }^{U} g \otimes f\right) c_{U, V}
$$

(b) for any objects $U, V, W$ in $\mathcal{C}$ we have

$$
c_{U \otimes V, W}=\left(c_{U, V_{W}} \otimes 1\right)\left(1 \otimes c_{V, W}\right) ;
$$

(c) for any objects $U, V, W$ in $\mathcal{C}$ we have

$$
c_{U, V \otimes W}=\left(1 \otimes c_{U, W}\right)\left(c_{U, V} \otimes 1\right) ;
$$

(d) the action of $\pi$ on $\mathcal{C}$ preserves the braiding, i.e., for any $\alpha \in \pi$ and any $V, W \in \mathcal{C}$ we have

$$
\varphi_{\alpha}\left(c_{V, W}\right)=c_{\varphi_{\alpha}(V), \varphi_{\alpha}(W)} .
$$

A crossed $\pi$-category equipped with a braiding is called a braided crossed $\pi$-category. A braided crossed $\pi$-category $\mathcal{C}$ is called balanced if it is equipped with a natural family of invertible morphisms $\theta_{U}: U \rightarrow{ }^{U} U$ (called twist) satisfying the following conditions:

(1) $\theta_{I}=\mathrm{id}_{I}: I \rightarrow I$

(2) for any object $U, V$ in $\mathcal{C}$ we have

$$
\theta_{U \otimes V}=c_{(U V) V, U U} c_{(U U),\left({ }^{V} V\right)}\left(\theta_{U} \otimes \theta_{V}\right)
$$

(3) the action of $\pi$ on $\mathcal{C}$ preserves the twist, i.e., for any $\alpha \in \pi$ and any $U \in \mathcal{C}$ we have $\varphi_{\alpha}\left(\theta_{U}\right)=\theta_{\varphi_{\alpha}(U)}$.

A braided crossed $\pi$-category $\mathcal{C}$ is called tortile if it is balanced and each object $U$ has a dual $U^{*}$ such that $\theta_{U^{*}}=\left(\theta_{U}\right)^{*}$. 


\section{Tortile Yang-Baxter operators in crossed group-categories}

In this section we consider Yang-Baxter operators and twists in a crossed $\pi$-category $\mathcal{C}$. When $\pi=1$, one can define a Yang-Baxter operator on each object $U$ in $\mathcal{C}$ without a twist. However, for a general crossed $\pi$-category $\mathcal{C}$, one must define a twist first, then proceed to define a balanced Yang-Baxter operator by using the twist.

Definition 4 A twist on an object $U$ of a crossed $\pi$-category $\mathcal{C}$ is an invertible arrow $z: U \rightarrow{ }^{U} U$. A balanced Yang-Baxter operator on an object $U$ is an invertible arrow $y: U \otimes U \rightarrow{ }^{U} U \otimes U$ satisfying the hexagonal condition

$$
\left({ }^{U} y \otimes 1\right)(1 \otimes y)(y \otimes 1)=(1 \otimes y)\left(y^{\prime} \otimes 1\right)(1 \otimes y)
$$

where $y^{\prime}=\left({ }^{U} z \otimes 1\right) y\left(1 \otimes z^{-1}\right)$.

A left dual for an object $U$ of $\mathcal{C}_{\alpha}$ is an object $U^{*}$ in $\mathcal{C}_{\alpha^{-1}}$ together with arrows

$$
b_{U}: I \rightarrow U \otimes U^{*} \quad \text { and } \quad d_{U}: U^{*} \otimes U \rightarrow I
$$

such that

$$
\left(d_{U} \otimes 1\right)\left(1 \otimes b_{U}\right)=1 \quad \text { and } \quad\left(1 \otimes d_{U}\right)\left(b_{U} \otimes 1\right)=1 .
$$

If both $U, V$ have duals, then each arrow $f: U \rightarrow V$ gives rise to an arrow

$$
f^{*}:\left(d_{V} \otimes 1\right)(1 \otimes f \otimes 1)\left(1 \otimes b_{U}\right): V^{*} \rightarrow U^{*} .
$$

A balanced Yantg-Baxter operator on an object $U \in \mathcal{C}$ is called dualizable if $U$ has a dual and, both the arrows $u:{ }^{U} U^{*} \otimes U \rightarrow U \otimes U^{*}$ and $v: U^{*} \otimes$ $U \rightarrow U^{*} U \otimes U^{*}$, given by the equations

$$
u=\left(d_{U_{U}} \otimes 1 \otimes 1\right)(1 \otimes y \otimes 1)\left(1 \otimes 1 \otimes b_{U}\right)
$$

and

$$
\begin{aligned}
v= & \left(d_{U} \otimes 1 \otimes 1\right)\left(1 \otimes 1 \otimes{ }^{*} z^{-1} \otimes 1\right)\left(1 \otimes y^{-1} \otimes 1\right) \\
& (1 \otimes z \otimes 1 \otimes 1)\left(1 \otimes 1 \otimes b_{U}\right)
\end{aligned}
$$

are invertible. A balanced Yang-Baxter operator on an object $U$ is called tortile if it is dualizable and the following identity holds.

$$
U_{z z}=\left(1 \otimes d_{U}\right)\left(1 \otimes{ }^{U} v^{-1}\right)\left(y^{\prime} \otimes 1\right)\left(1 \otimes b_{U}\right): U \rightarrow{ }^{U}\left({ }^{U} U\right) .
$$


In a balanced crossed $\pi$-category $\mathcal{C}$, we have a balanced YB-operator $\left(y=c_{U, U}, z=\theta_{U}\right)$ on each object $U$. If $U$ has a dual, then we have the identities $u=c_{U, U^{*}}^{-1}$ and $v=c_{U^{*}, U}$ in $\mathcal{C}$. Hence $\left(y=c_{U, U}, z=\theta_{U}\right)$ is dualizable. The next proposition shows that a balanced crossed $\pi$-category $\mathcal{C}$ becomes a tortile crossed $\pi$-category iff the above balanced YB-operators $\left(y=c_{U, U}, z=\theta_{U}\right)$ become tortile for all objects $U$ in $\mathcal{C}$.

Proposition 1 In a balanced crossed $\pi$-category $\mathcal{C}$, if $U$ is an object with a dual $U^{*}$, then the pair $\left(c_{U, U}, \theta_{U}\right)$ is a tortile $Y B$-operator iff $\theta_{U_{U^{*}}}=\left(\theta_{U}\right)^{*}$.

Proof. We first observe that if $\left(U^{*}, d_{U}, b_{U}\right)$ is a dual for $U$, then $\left({ }^{U} U, d_{U}{ }^{c_{U}} U_{U^{*}}, c_{U^{*}, U_{U}}^{-1} b_{U}\right)$ is a dual for $U^{*}$. Then for an arrow $f: U^{*} \rightarrow V^{*}$ in $\mathcal{C}$, we obtain an arrow

$$
f^{\sharp}=\left(1 \otimes d_{V_{V}}\right)\left(1 \otimes{ }^{U} f \otimes 1\right)\left(b_{U} \otimes 1\right):{ }^{V} V \rightarrow{ }^{U} U .
$$

Applying this construction to the arrow $\theta_{U_{U^{*}}}:{ }^{U} U^{*} \rightarrow U^{*}$, we see that

$$
\begin{aligned}
& \left(\theta_{U_{U}}\right)^{\sharp} \theta_{U} \\
& =\left(d_{U_{U}} \otimes 1\right)\left(c_{U_{U}, U_{U^{*}}} \otimes 1\right)\left(1 \otimes \theta_{U_{U^{*}}} \otimes 1\right)\left(1 \otimes c_{U_{U^{*}, U}\left({ }_{U} U\right)}^{-1}\right)\left(1 \otimes b_{U}\right) \theta_{U} \\
& =\left(d_{U} \otimes 1\right)\left(c_{U_{U}, U_{U^{*}}} \otimes 1\right)\left(1 \otimes \theta_{U_{U^{*}}} \otimes 1\right)\left(\theta_{U} \otimes 1 \otimes 1\right) \\
& \left(1 \otimes c_{U^{*},{ }^{U}\left({ }_{U}\right)}^{-1}\right)\left(1 \otimes b_{U}\right) \\
& =\left(d_{U_{U}} \otimes 1\right)\left(c_{U_{U}, U_{U^{*}}} \otimes 1\right)\left(\theta_{U} \otimes \theta_{U_{U^{*}}} \otimes 1\right)\left(1 \otimes c_{U_{U^{*},}\left({ }_{U} U\right)}^{-1}\right)\left(1 \otimes b_{U}\right) \\
& =\left(d_{U_{U}} \otimes 1\right)\left(\theta_{U_{U^{*}}} \otimes \theta_{U_{U}} \otimes 1\right)\left(c_{\left.U_{U^{*}, U_{U}}^{-1} \otimes 1\right)\left(1 \otimes c_{U^{*}, U}^{-1}\left({ }_{U}\right)\right.}\right)\left(1 \otimes b_{U}\right) \\
& =\left(d_{U_{U}} \otimes 1\right)\left(c_{U_{U^{*}, U}}^{-1} \otimes 1\right)\left(1 \otimes c_{U_{U^{*}, U}\left({ }_{U}\right)}^{-1}\right)\left(1 \otimes b_{U}\right) \\
& =\left(d_{U_{U}} \otimes 1\right) c_{U^{*},{ }_{U} \otimes^{U}\left({ }_{U} U\right)}^{-1}\left(1 \otimes b_{U}\right) \\
& =\left(1 \otimes d_{U}\right) c_{\left.U_{U^{*} \otimes} U_{U} U_{(}{ }^{U} U\right)} c_{U_{U^{*}, U} U_{U \otimes}\left({ }_{U} U\right)}^{-1}\left(1 \otimes b_{U}\right)
\end{aligned}
$$

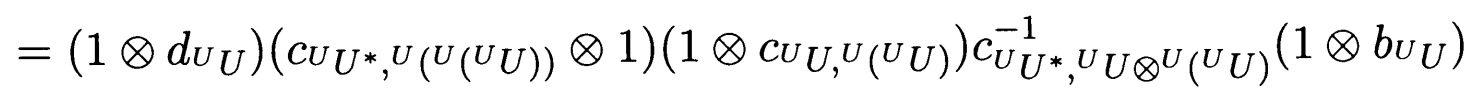

$$
\begin{aligned}
& =\left(1 \otimes d_{U}\right)\left(c_{U_{U^{*}, U}\left(U_{(U}\left({ }_{U}\right)\right)} \otimes 1\right) c_{U_{U^{*}, U}\left(U_{(U} U_{U}\right) \otimes^{U} U}\left(c_{U^{,}{ }_{U}} \otimes 1\right)\left(1 \otimes b_{U}\right) \\
& =\left(1 \otimes d_{U}\right)\left(1 \otimes c_{U_{U^{*},{ }_{U}}}^{-1}\right)\left(c_{U^{U} U_{U}} \otimes 1\right)\left(1 \otimes b_{U}\right) \\
& =\left(1 \otimes d_{U}\right)\left(1 \otimes{ }^{U} v^{-1}\right)\left(y^{\prime} \otimes 1\right)\left(1 \otimes b_{U}\right) .
\end{aligned}
$$

Thus the balanced YB-operator $(y, z)$ is tortile iff $\left(\theta_{U_{U}}\right)^{\sharp} \theta_{U}={ }^{U} z z=$ ${ }^{U} \theta_{U} \theta_{U}$. That is, $\left(\theta_{U_{U}}\right)^{\sharp}={ }^{U} \theta_{U}$. Then it is easy to see that the condition $\left(\theta_{U_{U}}\right)^{\sharp}={ }^{U} \theta_{U}$ is equivalent to the relation $\theta_{U_{U^{*}}}=\left(\theta_{U}\right)^{*}$. 
Definition 5 For a crossed $\pi$-category $\mathcal{C}$, the centralizer $\mathcal{L}_{\mathcal{C}}(U)$ of an object $U \in \mathcal{C}$ is the category whose objects are pairs $(X, \alpha)$ where $X$ is an object in $\mathcal{C}$ and $\alpha: U \otimes X \rightarrow{ }^{U} X \otimes U$ is an isomorphism in $\mathcal{C}$, and whose arrows $f:(X, \alpha) \rightarrow(Y, \beta)$ are the arrows $f: X \rightarrow Y$ in $\mathcal{C}$ such that $\beta(1 \otimes$ $f)=\left({ }^{U} f \otimes 1\right) \alpha$.

Then $\mathcal{L}_{\mathcal{C}}(U)$ becomes a $\pi$-category by the rule $(X, \alpha) \in \mathcal{C}_{x} \Leftrightarrow X \in \mathcal{C}_{x}$ for $x \in \pi$ and the tensor product $(X, \alpha) \otimes(Y, \beta)=(X \otimes Y,(\alpha \otimes 1)(1 \otimes \beta))$. Similarly, one can define the centralizer $\mathcal{L}_{\mathcal{C}}(h)$ of an arrow $h: U \rightarrow V$ in $\mathcal{C}$ as follows. The objects of $\mathcal{L}_{\mathcal{C}}(h)$ are triples $(X, \alpha, \beta)$ where $X$ is an object in $\mathcal{C}$ and $\alpha: U \otimes X \rightarrow{ }^{U} X \otimes U$ and $\beta: V \otimes X \rightarrow{ }^{V} X \otimes V$ are isomorphisms in $\mathcal{C}$ such that $\beta(h \otimes 1)=(1 \otimes h) \alpha$. The arrows $(X, \alpha, \beta) \rightarrow(Y, \gamma, \delta)$ in $\mathcal{L}_{\mathcal{C}}(h)$ are arrows $f: X \rightarrow Y$ in $\mathcal{C}$ such that $\left({ }^{U} f \otimes 1\right) \alpha=\gamma(1 \otimes f)$ and $\left({ }^{V} f \otimes\right.$ 1) $\beta=\delta(1 \otimes f)$. This category $\mathcal{L}_{\mathcal{C}}(h)$ also admits a $\pi$-category structure.

Definition 6 A crossed $\pi$-category $\mathcal{C}$ is called connected if for each pair $(X, Y)$ of objects, there exists an invertible arrow $g(X, Y):{ }^{X} Y \rightarrow Y$ such that $g(I, Y)=\operatorname{id}_{Y}, g(X, I)=\operatorname{id}_{I}, g\left(X^{\prime} \otimes X, Y\right)=g\left(X^{\prime}, Y\right){ }^{X^{\prime}} g(X, Y)$ and $g\left(X, Y \otimes Y^{\prime}\right)=g(X, Y) \otimes g\left(X, Y^{\prime}\right)$.

When a crossed $\pi$-category $\mathcal{C}$ is connected, the categories $\mathcal{L}_{\mathcal{C}}(U)$ and $\mathcal{L}_{\mathcal{C}}(h)$ become crossed $\pi$-categories via ${ }^{(X, \alpha)}(Y, \beta)=\left({ }^{X} Y,\left({ }^{U} g^{-1}(X, Y) \otimes\right.\right.$ 1) $\beta(1 \otimes g(X, Y)))$ and ${ }^{(X, \alpha, \beta)}(Y, \gamma, \delta)=\left({ }^{X} Y,\left({ }^{U} g^{-1}(X, Y) \otimes 1\right) \gamma(1 \otimes g(X, Y))\right.$, $\left.\left({ }^{U} g^{-1}(X, Y) \otimes 1\right) \delta(1 \otimes g(X, Y))\right)$.

Definition 7 For a crossed $\pi$-category $\mathcal{C}$, the center $\mathcal{L}_{\mathcal{C}}$ of $\mathcal{C}$ is the category whose objects are pairs $(U, \alpha)$ where $U \in \mathcal{C}$ and $\alpha: U \otimes-\rightarrow^{U}-\otimes U$ is a natural isomorphism obeying the following two conditions:

(1) $\alpha_{I}=1$;

(2) $\alpha_{X \otimes Y}=\left(1 \otimes \alpha_{Y}\right)\left(\alpha_{X} \otimes 1\right)$ for all $X, Y \in \mathcal{C}$.

An arrow $f:(U, \alpha) \rightarrow(V, \beta)$ in $\mathcal{L}_{\mathcal{C}}$ is an arrow $f: U \rightarrow V$ in $\mathcal{C}$ such that $\beta_{X}(f \otimes 1)=(1 \otimes f) \alpha_{X}$ for all $X \in \mathcal{C}$.

Then $\mathcal{L}_{\mathcal{C}}$ becomes a crossed $\pi$-category with $(U, \alpha) \otimes(V, \beta)=(U \otimes$ $V,(\alpha \otimes 1)(1 \otimes \beta))$ and ${ }^{(U, \alpha)}(V, \beta)=\left({ }^{U} V,{ }^{U} \beta_{U^{*} X}\right)$.

Proposition 2 (a) For a crossed $\pi$-category $\mathcal{C}$, the crossed $\pi$-category $\mathcal{L}_{\mathcal{C}}$ is braided via $\alpha_{V}:(U, \alpha) \otimes(V, \beta) \rightarrow{ }^{(U, \alpha)}(V, \beta) \otimes(U, \alpha)$. 
(b) Let $\mathcal{C}$ be a crossed $\pi$-category. Then for each object $U \in \mathcal{C}$, the equation $F(X)=\left(X, \alpha_{X}\right)$ determines a bijection between objects $(U, \alpha) \in$ $\mathcal{L}_{\mathcal{C}}$ and tensor functors $F: \mathcal{C} \rightarrow \mathcal{L}_{\mathcal{C}}(U)$. Similarly, for each arrow $h: U \rightarrow$ $V$ in $\mathcal{C}$, the equation $F^{\prime}(X)=\left(X, \alpha_{X}, \beta_{X}\right)$ determines a bijection between arrows $h:(U, \alpha) \rightarrow(V, \beta) \in \mathcal{L}_{\mathcal{C}}$ and tensor functors $F^{\prime}: \mathcal{C} \rightarrow \mathcal{L}_{\mathcal{C}}(h)$.

(c) For a crossed $\pi$-category $\mathcal{C}$, the equation $G(U)=\left(U, c_{U,-}\right)$ determines a bijection between braidings $c$ on $\mathcal{C}$ and crossed $\pi$-functors $G: \mathcal{C} \rightarrow$ $\mathcal{L}_{\mathcal{C}}$

Proof. Straightforward.

For a connected crossed $\pi$-category $\mathcal{C}$, let $(y, z)$ be a balanced YBoperator on an object $U$ such that $g(U, U)=z^{-1}$. Then we have the following lemma:

Lemma 1 (a) The balanced YB-operator $(y, z)$ defines a balanced $Y B$-operator on the object $(U, y) \in \mathcal{L}_{\mathcal{C}}(U)$. If $(y, z)$ is dualizable, then $\left(U^{*}, u^{-1}\right) \in \mathcal{L}_{\mathcal{C}}(U)$ is a left dual for the object $(U, y) \in \mathcal{L}_{\mathcal{C}}(U)$. Moreover, $(y, z)$ defines a dualizable balanced $Y B$-operator on $(U, y)$.

(b) The centralizer $\mathcal{L}_{\mathcal{C}}\left(U^{*}\right)$ contains $(U, v)$ and $\left(U^{*}, w\right)$ where $w=$ $\left(d_{U} \otimes 1 \otimes 1\right)\left(1 \otimes 1 \otimes U^{*} z^{*} \otimes 1\right)(1 \otimes u \otimes 1)\left(1 \otimes z^{*-1} \otimes 1 \otimes 1\right)\left(1 \otimes 1 \otimes b_{U}\right)$. The object $\left(U^{*}, w\right)$ is dual to $(U, v)$ and $(y, z)$ defines a balanced $Y B$-operator on $(U, v)$.

(c) If $(y, z)$ is a tortile Yang-Baxter operator, then it is also a tortile Yang-Baxter operator on $(U, y) \in \mathcal{L}_{\mathcal{C}}(U)$ and $(U, v) \in \mathcal{L}_{\mathcal{C}}\left(U^{*}\right)$.

Proof. (a) For arrows $(U, y) \rightarrow{ }^{(U, y)}(U, y)$ and $(U, y) \otimes(U, y) \rightarrow$ ${ }^{(U, y)}(U, y) \otimes(U, y)$ in $\mathcal{L}_{\mathcal{C}}(U)$, we take the arrows $z: U \rightarrow{ }^{U} U$ and $y:$ $U \otimes U \rightarrow{ }^{U} U \otimes U$ in $\mathcal{C}$. Then by the hexagnal condition on $(y, z)$ and the assumption $g(U, U)=z^{-1}$, we see that these arrows are indeed arrows in $\mathcal{L}_{\mathcal{C}}(U)$.

(b) We have to show that the object $\left(U^{*}, w\right)$ is dual to $(U, v)$. For this it is convenient to use a diagrammatic notion as used in [3], [5]. For example, the following equalities show that the arrow $d_{U}: U^{*} \otimes U \rightarrow I$ becomes an arrow $\left(U^{*}, w\right) \otimes(U, v) \rightarrow\left(I, \operatorname{id}_{U^{*}}\right)$ in $\mathcal{L}_{\mathcal{C}}\left(U^{*}\right)$. 

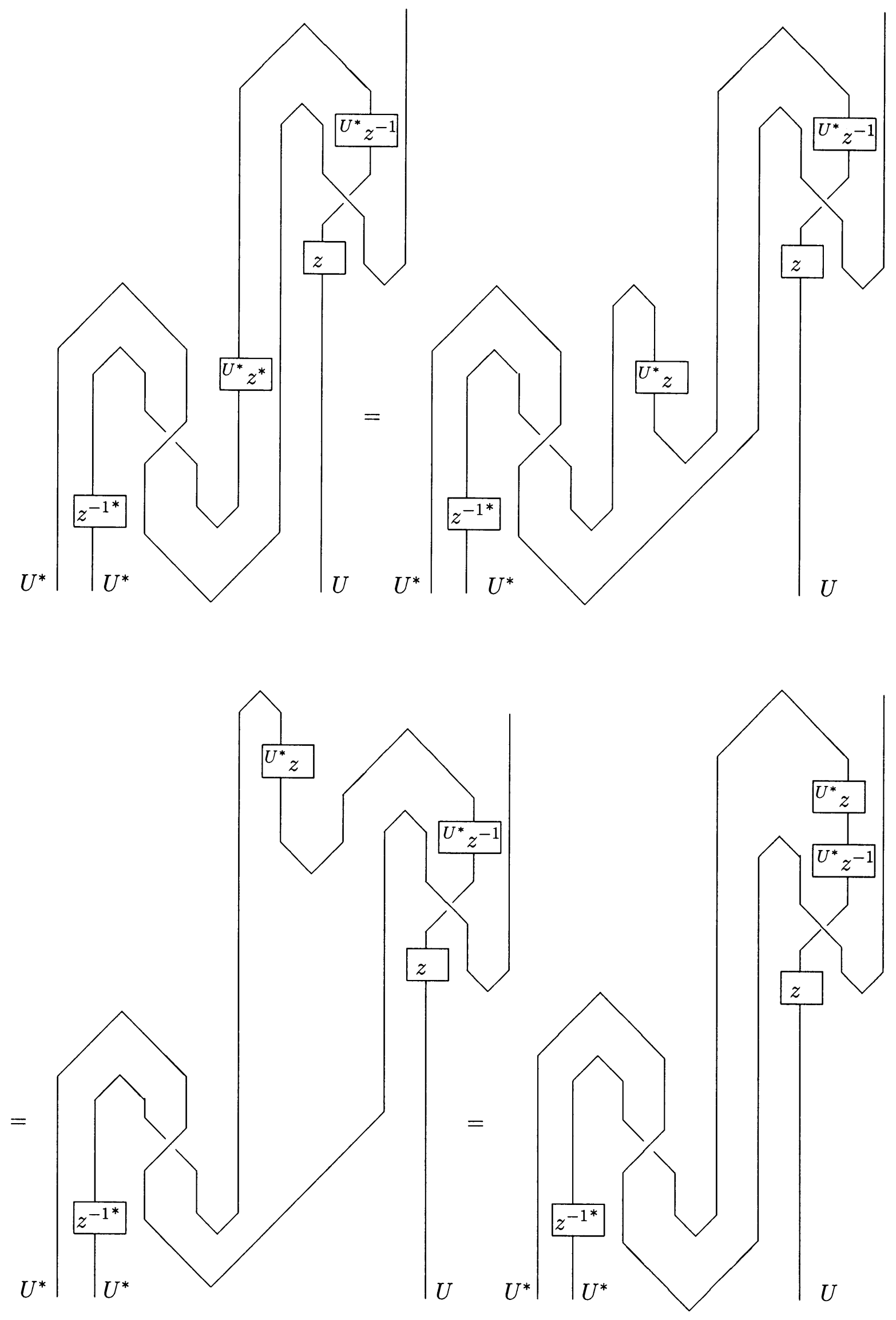

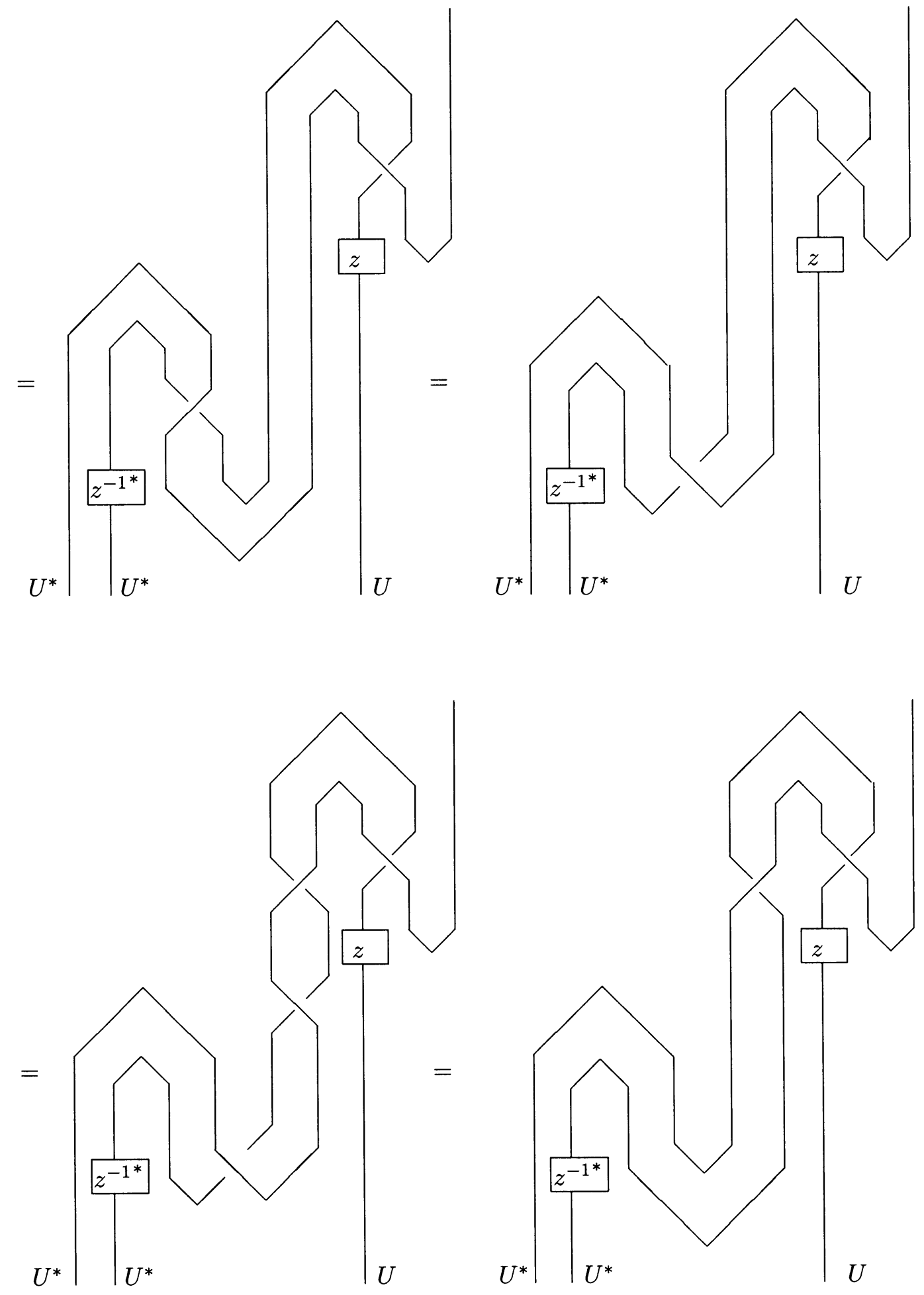

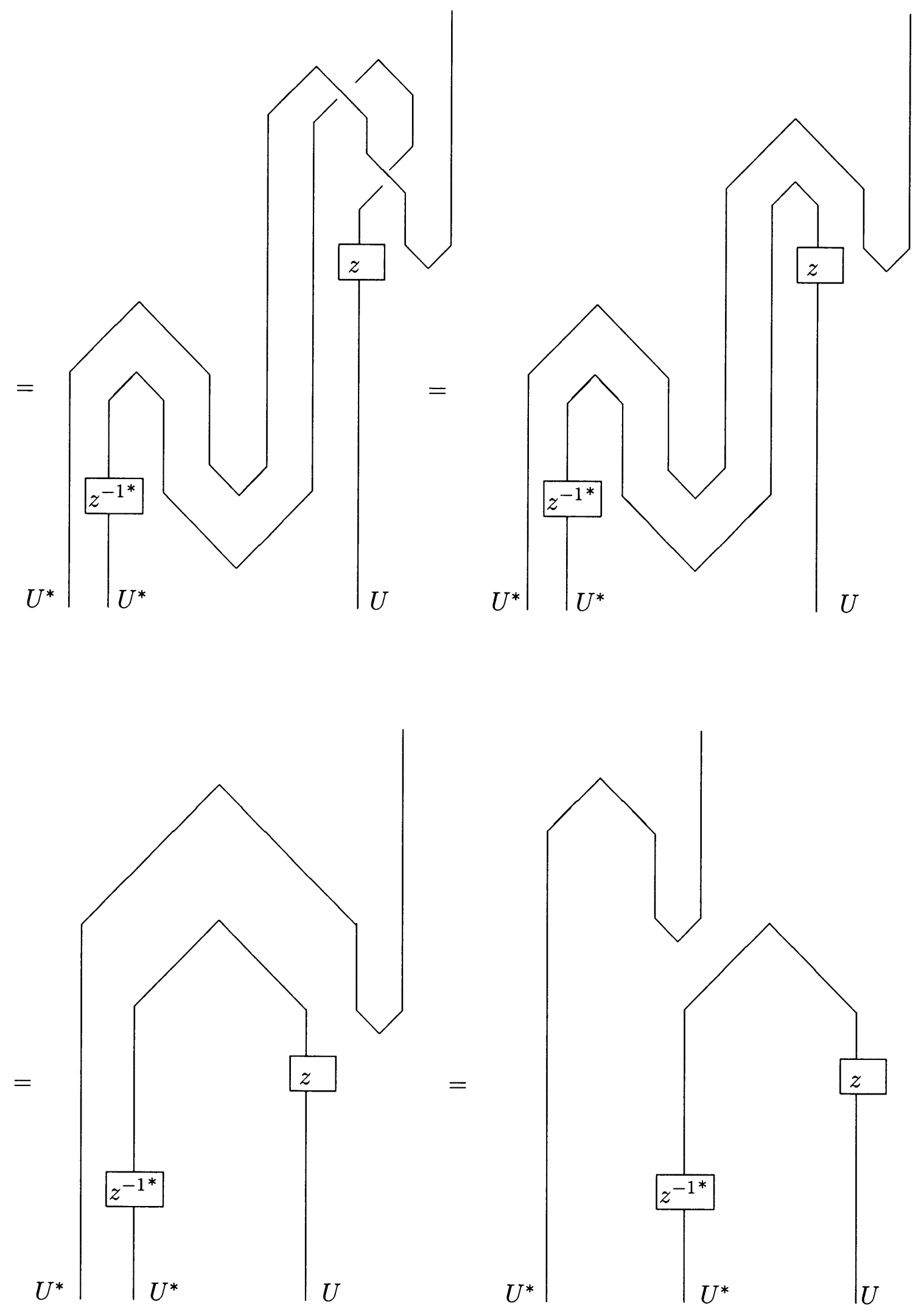


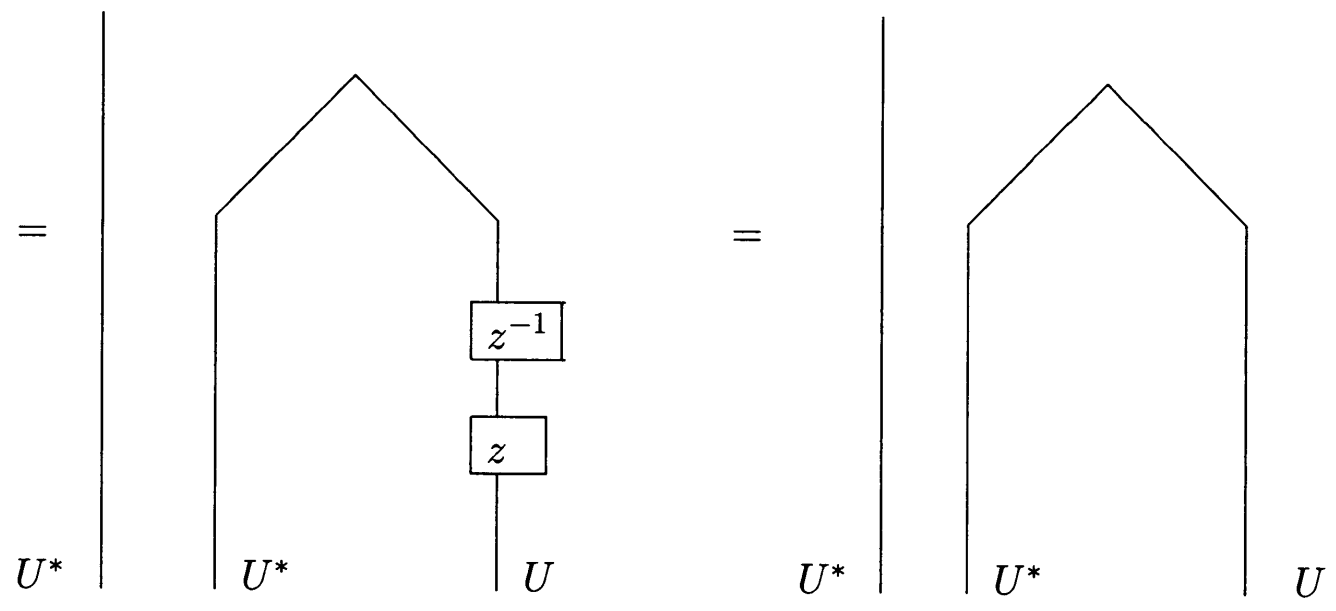

The next equalities show that the arrow $y: U \otimes U \rightarrow{ }^{U} U \otimes U$ defines an arrow $(U, v) \otimes(U, v) \rightarrow{ }^{(U, v)}(U, v) \otimes(U, v)$ in $\mathcal{L}_{\mathcal{C}}\left(U^{*}\right)$.

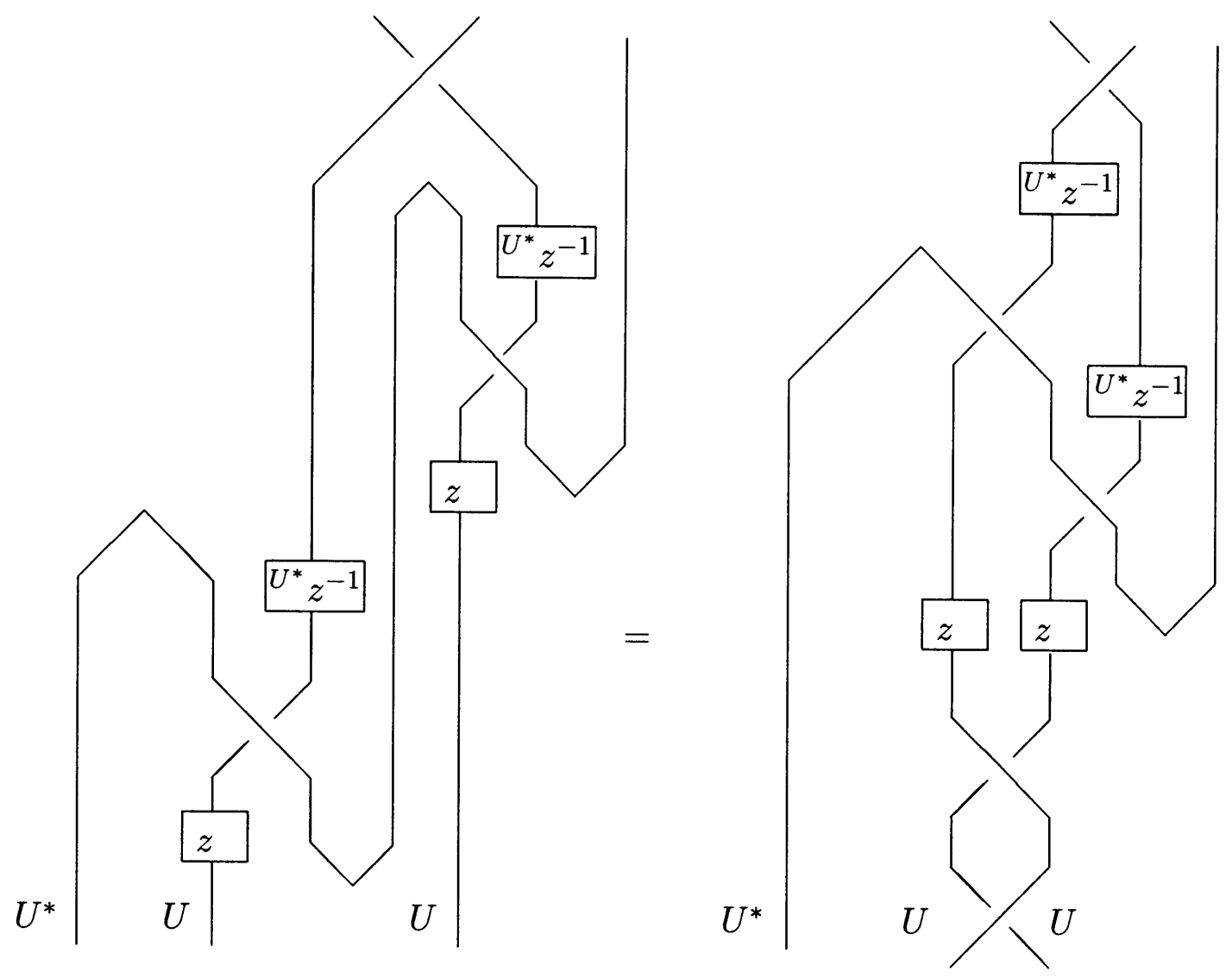



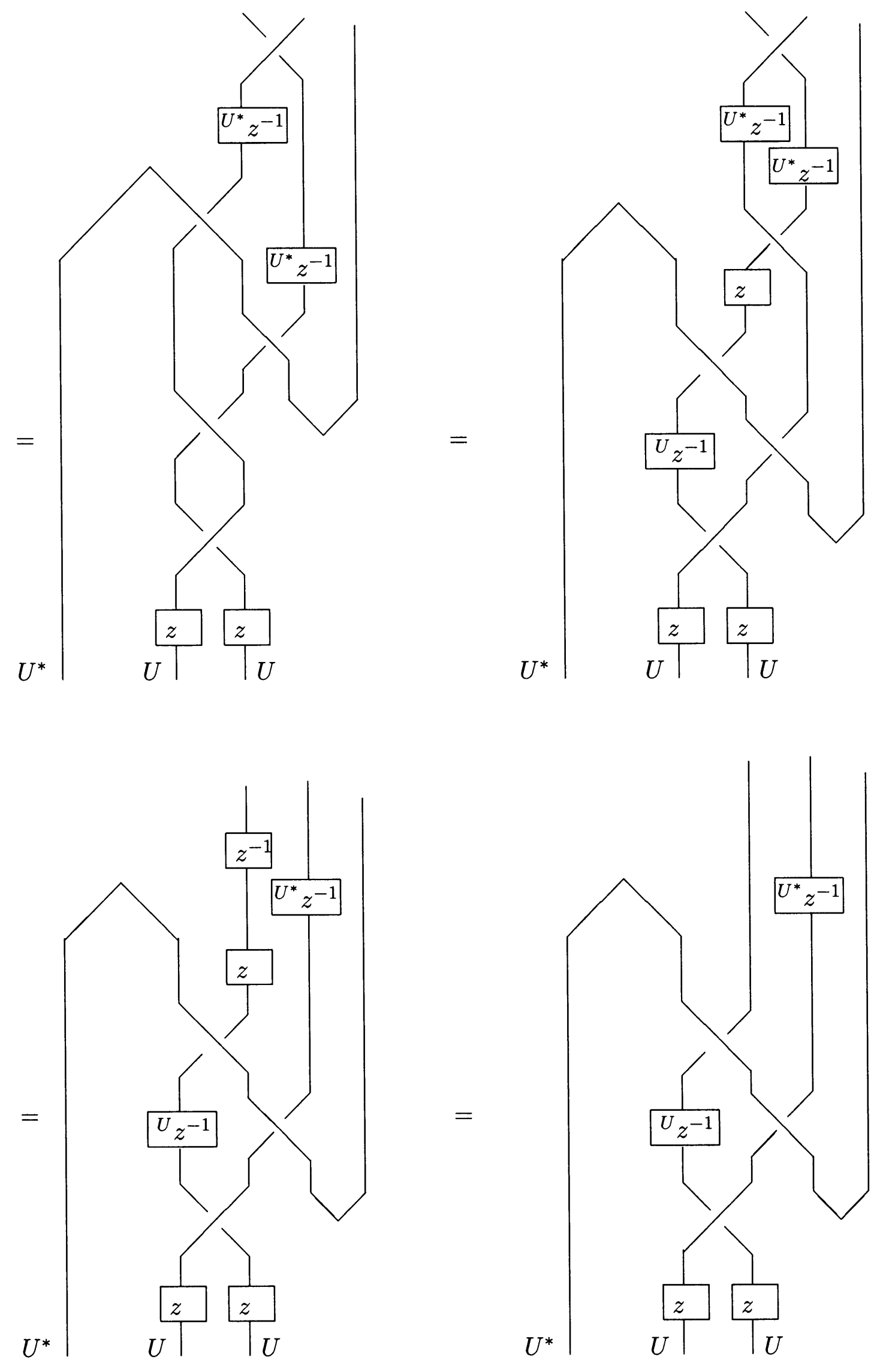

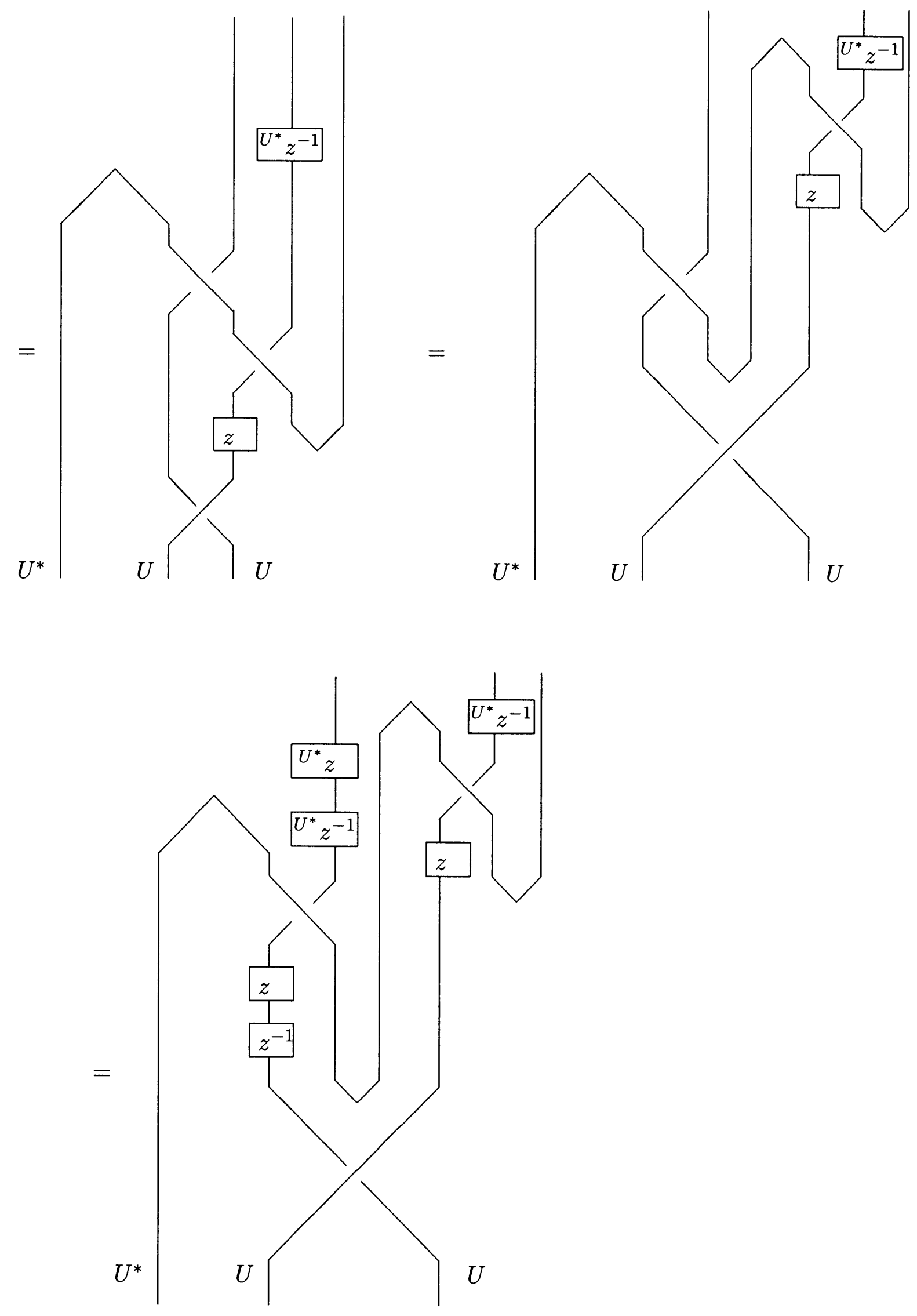
(c) Straightforward.

Let $\mathcal{F}$ be the crossed $\mathbf{Z}$-category freely generated by objects $X$ and $X^{*}$ and arrows $d_{X}: X^{*} \otimes X \rightarrow I, b_{X}: I \rightarrow X \otimes X^{*}, y: X \otimes X \rightarrow{ }^{X} X \otimes X$, $z: X \rightarrow^{X} X$, subject to the conditions that $X \in \mathcal{F}_{1}, X^{*} \in \mathcal{F}_{-1}, X^{*}$ is left dual to $X$ via $d_{X}$ and $b_{X}$, and $(y, z)$ is a tortile $Y B$-operator on $X$.

Now we state our main theorem in this paper.

Theorem The category $\mathcal{F}$ admits a unique braiding $c$ and a twist $\theta$ such that $c_{X, X}=y, \theta_{X}=z$ and $\theta_{X^{*}}={ }^{*}\left(\theta_{X}\right)^{*}$.

To prove the existence of such a braiding, we begin with the following proposition.

Proposition 3 There is a natural isomorphisms

$$
c_{X,-}: X \otimes-\rightarrow^{X}-\otimes X \quad \text { and } \quad c_{X^{*},-}: X^{*} \otimes-\rightarrow^{X^{*}}-\otimes X^{*}
$$

such that $\left(X, c_{X,-}\right)$ and $\left(X^{*}, c_{X^{*},-}\right) \in \mathcal{L}_{\mathcal{F}}$ where $c_{X, X}=y$ and $c_{X^{*}, X}=v$.

Proof. We first show that the category $\mathcal{F}$ is a connected crossed $\mathbf{Z}$-category. For this, observe that the category $\mathcal{F}$ is generated as a monoidal category by the objects ${ }^{n} X,{ }^{* n} X, X^{n} X^{*},{ }^{* n} X^{*}$, where $X^{n}$ and $X^{* n}$ mean $X \otimes$ $X \otimes \cdots \otimes X$ ( $n$ times $)$ and $X^{*} \otimes X^{*} \otimes \cdots \otimes X^{*}(n$ times $)$ respectively, and $n$ runs through the set of non-negative integers. Besides, the objects ${ }^{n} X$ and ${ }^{X^{* n}} X$ belong to the same class of $X$, and the objects ${ }^{X^{n}} X^{*}$ and ${ }^{X^{* n}} X^{*}$ belong to the same class of $X^{*}$. Using this property we can prove that for any object $Y$ there exists an isomorphism ${ }^{Y} X \rightarrow X$. Indeed, for any objects $Y$ and $Z$ we can define an isomophism ${ }^{Y \otimes Z} X \rightarrow X$ once we obtain isomorphisms $f:{ }^{Y} X \rightarrow X$ and $g:{ }^{Z} X \rightarrow X$ since we can use the composition $f^{Y} g$. Thus it is enough to show that there are isomorphisms ${ }^{X} X \rightarrow X$ and $X^{*} X \rightarrow X$, and we can take the arrows $z^{-1}$ and ${ }^{X^{*}} z$. The same argument applies to show that for any object $Y$ there exists an isomorphism $Y\left(X^{n} X\right) \rightarrow X^{n} X$. Actually, we only need isomorphisms $X\left(X^{n} X\right) \rightarrow{ }^{n} X$ and $X^{*}\left(X^{n} X\right) \rightarrow X^{n} X$, and we can take the arrows $X^{n} z^{-1}$ and $X^{n}\left(X^{*} z\right)$. Similarly, one can prove that for any object $Y$ there are isomorphisms $Y\left(X^{* n} X\right) \rightarrow{ }^{X^{* n}} X,{ }^{Y}\left(X^{n} X^{*}\right) \rightarrow{ }^{X^{n}} X^{*}$ and ${ }^{Y}\left(X^{* n} X^{*}\right) \rightarrow{ }^{X^{* n}} X^{*}$. Finally, we observe that for any object $Y, Z$ and $W$, an isomorphism ${ }^{Y}(Z \otimes W)=$ ${ }^{Y} Z \otimes{ }^{Y} W \rightarrow Z \otimes W$ is obtained from ${ }^{Y} Z \rightarrow Z$ amd ${ }^{Y} W \rightarrow W$. Hence the category $\mathcal{F}$ is connected, and the categories $\mathcal{L}_{\mathcal{F}}(X)$ and $\mathcal{L}_{\mathcal{F}}\left(X^{*}\right)$ become 
crossed Z-categories. Moreover, the condition $g(X, X)=z^{-1}$ is satisfied. Thus we can use the universality of the category $\mathcal{F}$ and Lemma 1 to get crossed Z-functors $\mathcal{F} \rightarrow \mathcal{L}_{\mathcal{F}}(X)$ and $\mathcal{F} \rightarrow \mathcal{L}_{\mathcal{F}}\left(X^{*}\right)$. In particular, these functors are tensor functors, hence by Proposition 2 (b) we obtain the natural isomorphisms $c_{X,-}: X \otimes-\rightarrow^{X}-\otimes X$ and $c_{X^{*},-}: X^{*} \otimes-\rightarrow^{X^{*}}-$ $\otimes X^{*}$.

Lemma 2 The pair $(y, z)$ becomes a tortile $Y B$-operator on the object $\left(X, c_{X,-}\right)$ in $\mathcal{L}_{\mathcal{F}}$. Also, the object $\left(X^{*}, c_{X^{*},-}\right)$ is left dual to the object $\left(X, c_{X,-}\right)$ in $\mathcal{L}_{\mathcal{F}}$

Proof. To obtain a tortile $Y B$-operator on the object $\left(X, c_{X,-}\right)$ in $\mathcal{L}_{\mathcal{F}}$, we first consider the centralizer $\mathcal{L}_{\mathcal{F}}(y)$ of $y: X \otimes X \rightarrow{ }^{X} X \otimes X$. Then we see that the category $\mathcal{L}_{\mathcal{F}}(y)$ contains the objects $(X, \alpha, \beta)$ and $\left(X^{*}, \gamma, \delta\right)$ where $\alpha=\left(y^{\prime} \otimes 1\right)(1 \otimes y), \beta=\left({ }^{X} y \otimes 1\right)(1 \otimes y), \gamma=\left({ }^{X} z^{*-1} \otimes 1\right)\left(u^{-1} \otimes\right.$ $1)\left(1 \otimes z^{*} \otimes 1\right)\left(1 \otimes u^{-1}\right), \delta=\left({ }^{X} u^{-1} \otimes 1\right)\left(1 \otimes u^{-1}\right)$. Moreover, the object $\left(X^{*}, \gamma, \delta\right)$ is dual to $(X, \alpha, \beta)$, and we obtain a tortile $Y B$-operator $(y, z)$ on $(X, \alpha, \beta)$ in $\mathcal{L}_{\mathcal{F}}(y)$. Since $\mathcal{L}_{\mathcal{F}}(y)$ is a crossed $\mathbf{Z}$-category, the universal property of $\mathcal{F}$ induces a crossed tensor functor $\mathcal{F} \rightarrow \mathcal{L}_{\mathcal{F}}(y)$, which is a section of the projection $\mathcal{L}_{\mathcal{F}}(y) \rightarrow \mathcal{F}$. In particular, this functor is a tensor functor, hence by Proposition 2 (b), we obtain an arrow $y:\left(X, c_{X,-}\right) \otimes$ $\left(X, c_{X,-}\right) \rightarrow^{\left(X, c_{X,-}\right)}\left(X, c_{X,-}\right) \otimes\left(X, c_{X,-}\right)$ in $\mathcal{L}_{\mathcal{F}}$.

Next consider the category $\mathcal{L}_{\mathcal{F}}(z)$. Then we see that the category $\mathcal{L}_{\mathcal{F}}(z)$ contains the objects $(X, y, \eta)$ and $\left(X^{*}, u^{-1}, \zeta\right)$ where $\eta=(1 \otimes z) y\left(z^{-1} \otimes 1\right)$ and $\zeta=(1 \otimes z) u^{-1}\left(z^{-1} \otimes 1\right)$. In addition, $\left(X^{*}, u^{-1}, \zeta\right)$ is dual to $(X, y, \eta)$, and we obtain a tortile $Y B$-operator $(y, z)$ on the objects $(X, y, \eta)$ in $\mathcal{L}_{\mathcal{F}}(z)$. Thus we have a crossed tensor functor $\mathcal{F} \rightarrow \mathcal{L}_{\mathcal{F}}(z)$, which corresponds to an arrow $z:\left(X, c_{X,-}\right) \rightarrow^{\left(X, c_{X,-}\right)}\left(X, c_{X,-}\right)$ in $\mathcal{L}_{\mathcal{F}}$.

Then it is easy to see that the arrows $y:\left(X, c_{X,-}\right) \otimes\left(X, c_{X,-}\right) \rightarrow$ $\left(X, c_{X,-}\right)\left(X, c_{X,-}\right) \otimes\left(X, c_{X,-}\right)$ and $z:\left(X, c_{X,-}\right) \rightarrow\left(X, c_{X,-}\right)\left(X, c_{X,-}\right)$ in $\mathcal{L}_{\mathcal{F}}$ define a tortile $Y B$-operator on the object $\left(X, c_{X,-}\right)$ in $\mathcal{L}_{\mathcal{F}}$.

Finally, we check that the object $\left(X^{*}, c_{X^{*},-}\right)$ is left dual to the object $\left(X, c_{X,-}\right)$ in $\mathcal{L}_{\mathcal{F}}$. For this we consider the centralizers $\mathcal{L}_{\mathcal{F}}\left(d_{X}\right)$ and $\mathcal{L}_{\mathcal{F}}\left(b_{X}\right)$. We see that the category $\mathcal{L}_{\mathcal{F}}\left(d_{X}\right)$ contains the objects $\left(X, \tau, \operatorname{id}_{X}\right)$ and $\left(X^{*}, \sigma, \operatorname{id}_{X^{*}}\right)$ where $\tau=\left({ }^{X^{*}} z \otimes 1 \otimes 1\right)(v \otimes 1)\left(1 \otimes z^{-1} \otimes 1\right)(1 \otimes y)$ and $\sigma=$ $\left(X^{*} z^{*-1} \otimes 1 \otimes 1\right)(w \otimes 1)\left(1 \otimes z^{*} \otimes 1\right)\left(1 \otimes u^{-1}\right)$. Moreover, the object $\left(X^{*}, \sigma, \mathrm{id}_{X^{*}}\right)$ is dual to $\left(X, \tau, \operatorname{id}_{X}\right)$, and $(y, z)$ defines a tortile $Y B$-operator on the object $\left(X, \tau, \operatorname{id}_{X}\right)$ in $\mathcal{L}_{\mathcal{F}}\left(d_{X}\right)$. Thus we obtain a crossed tensor functor $\mathcal{F} \rightarrow$ 
$\mathcal{L}_{\mathcal{F}}\left(d_{X}\right)$, which corresponds to an arrow $\left(X^{*}, c_{X^{*},-}\right) \otimes\left(X, c_{X,-}\right) \rightarrow(I$, id $)$ in $\mathcal{L}_{\mathcal{F}}$. Similarly, the category $\mathcal{L}_{\mathcal{F}}\left(b_{X}\right)$ contains the objects $\left(X, \mu, \operatorname{id}_{X}\right)$ and $\left(X^{*}, \nu\right.$, id $\left._{X^{*}}\right)$ where $\mu=\left(z^{-1} \otimes 1 \otimes 1\right)(y \otimes 1)\left(1 \otimes{ }^{*} z \otimes 1\right)(1 \otimes v)$ and $\nu=$ $\left(z^{*} \otimes 1 \otimes 1\right)\left(u^{-1} \otimes 1\right)\left(1 \otimes X^{*} z^{*-1} \otimes 1\right)(1 \otimes w)$, which induces a crossed tensor functor $\mathcal{F} \rightarrow \mathcal{L}_{\mathcal{F}}\left(b_{X}\right)$ and hence an arrow $(I$, id $) \rightarrow\left(X, c_{X,-}\right) \otimes\left(X^{*}, c_{X^{*},-}\right)$ in $\mathcal{L}_{\mathcal{F}}$.

Using the lemma above, we can prove the existence of the braiding in Theorem. In fact, since $(y, z)$ is a tortile $Y B$-operator on the object $\left(X, c_{X,-}\right)$, and $\left(X^{*}, c_{X^{*},-}\right)$ is dual to the object $\left(X, c_{X,-}\right)$, we obtain a crossed tensor functor $\mathcal{F} \rightarrow \mathcal{L}_{\mathcal{F}}$. Thus by Proposition 2 (c), we see that $\mathcal{F}$ has a braiding $c$ with $c_{X, X}=y$.

Next we consider the existence of a twist $\theta$ in $\mathcal{F}$. For any braided crossed $\pi$-category $\mathcal{C}$, let $\mathcal{C}^{\prime}$ be the category whose objects are pairs $(U, \xi)$ where $\xi: U \rightarrow{ }^{U} U$ is an isomorphism in $\mathcal{C}$, and whose arrows $f:(U, \xi) \rightarrow(V, \zeta)$ are arrows $f: U \rightarrow V$ in $\mathcal{C}$ such that ${ }^{U} f \xi=\zeta f$. We can define a tensor product and a cross action on $\mathcal{C}^{\prime}$ by putting

$$
(U, \xi) \otimes(V, \zeta)=(U \otimes V, \chi) \text { and }{ }^{(U, \xi)}(V, \zeta)=\left({ }^{U} V,{ }^{U} \zeta\right)
$$

where $\chi={ }^{C_{U}\left({ }^{V} V\right),{ }^{U} U^{C}{ }_{U}{ }_{U},{ }_{V}}(\xi \otimes \zeta)$. This tensor product makes $\mathcal{C}^{\prime}$ into a crossed $\pi$-category. Applying this procedure to the braided crossed $\mathbf{Z}$ category $\mathcal{F}$, we obtain a crossed $\mathbf{Z}$-category $\mathcal{F}^{\prime}$. To get a left dual for the object $(X, z)$ in $\mathcal{F}^{\prime}$, we use the following lemma:

Lemma 3 The following identity holds in $\mathcal{F}$.

$$
\begin{aligned}
& z^{X^{*}} z\left(d_{X_{X}} \otimes 1\right)\left(1 \otimes c_{X_{X}, X^{*} X}^{-1}\right)\left(c_{X, X^{*}} \otimes 1\right)\left(b_{X} \otimes 1\right) \\
& \quad=1:{ }^{X} X \rightarrow{ }^{X} X .
\end{aligned}
$$

Proof. Since $z$ is a tortile $Y B$-operator the term $z^{X^{*}} z$ coincides with the composition $\left(1 \otimes d_{X}\right)\left(1 \otimes c_{X^{*}, X}^{-1}\right)\left(c_{X^{*}} X_{, X} \otimes 1\right) b_{X}$. Then the following equalities show the identity in Lemma 3 . 
Tortile Yang-Baxter operators for crossed group-categories

215
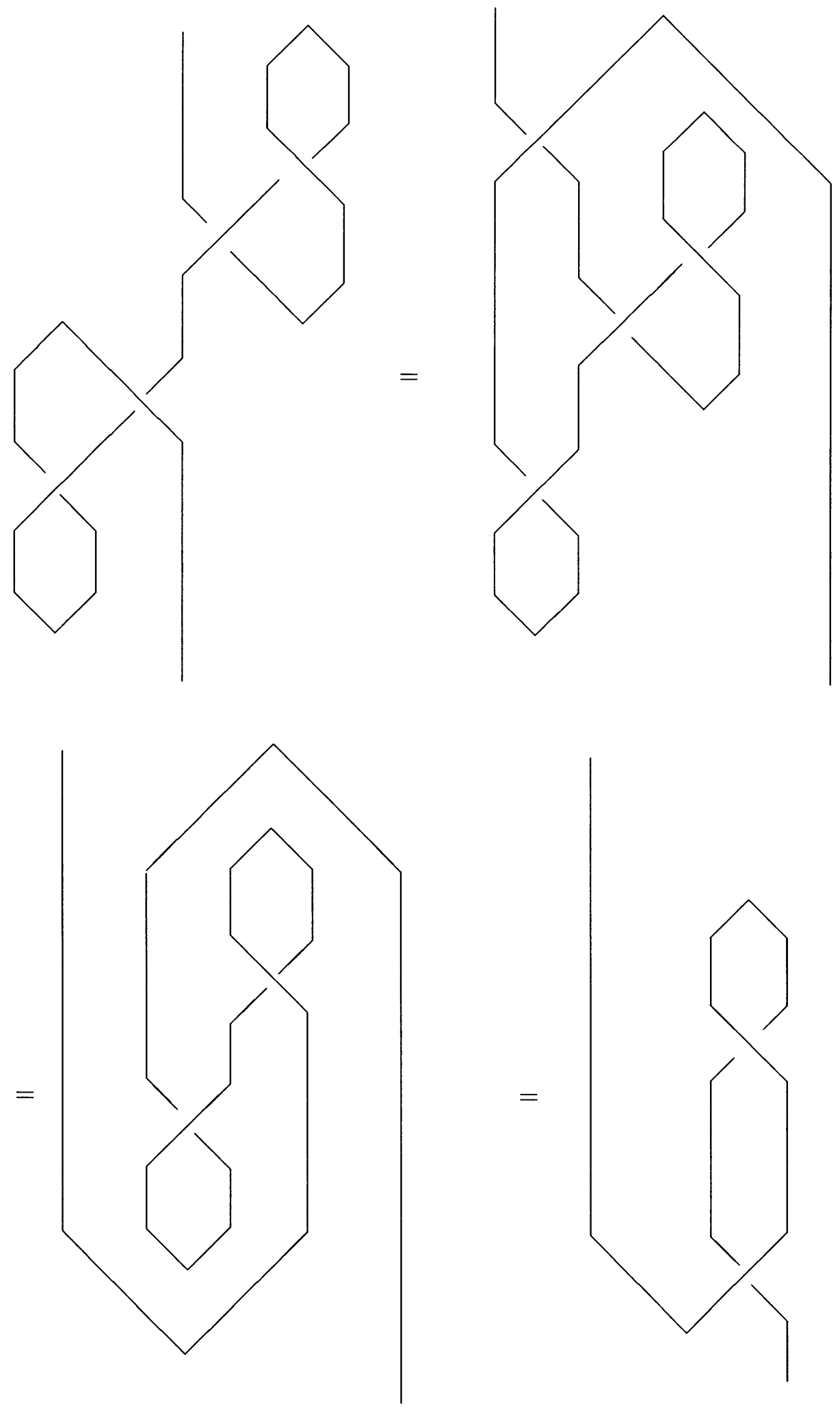


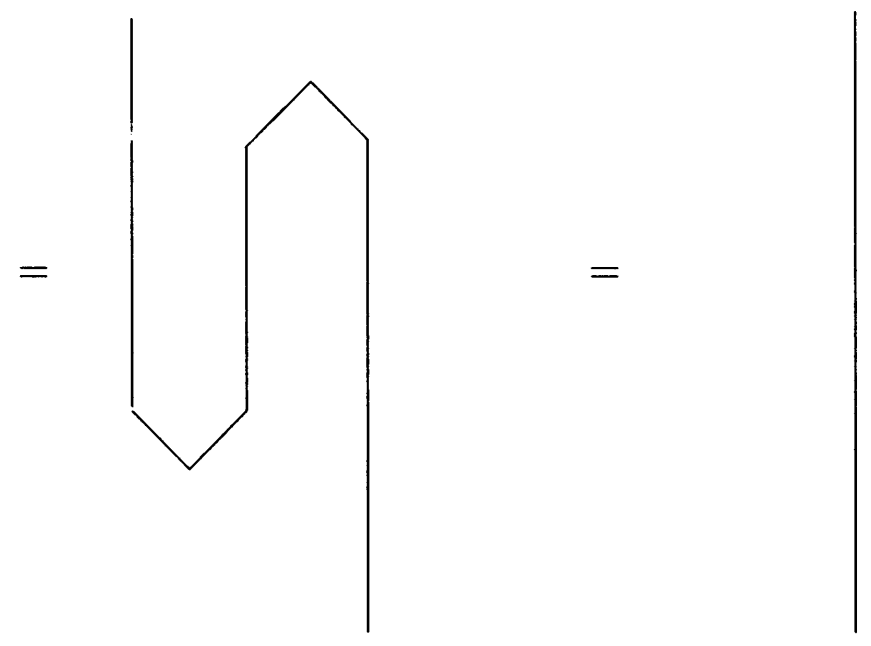

Using the lemma above, we can prove the following one:

Lemma 4 The object $\left(X^{*}, X^{*} z^{*}\right)$ is dual to the object $(X, z)$ in $\mathcal{F}^{\prime}$, and $(y, z)$ defines a tortile $Y B$-operator on $(X, z)$.

Proof. We have to show that the arrows $d_{X}: X^{*} \otimes X \rightarrow I$ and $b_{X}: I \rightarrow$ $X \otimes X^{*}$ define arrows $\left(X^{*}, X^{*} z^{*}\right) \otimes(X, z) \rightarrow(I$, id $)$ and $(I$, id $) \rightarrow(X, z) \otimes$ $\left(X^{*}, X^{*} z^{*}\right)$ in $\mathcal{F}^{\prime}$. The next caluculations show that the arrow $d_{X}: X^{*} \otimes$ $X \rightarrow I$ is an arrow $\left(X^{*}, X^{*} z^{*}\right) \otimes(X, z) \rightarrow(I$, id $)$ in $\mathcal{F}^{\prime}$.

$$
\begin{aligned}
& d_{X}\left(c_{X, X^{*} X^{*}}\right)\left(c_{X^{*} X^{*},{ }_{X}}\right)\left({ }^{X^{*}} z^{*} \otimes z\right) \\
& =d_{X} c_{X, X^{*} X^{*}}\left(d_{X^{*} X} \otimes 1 \otimes 1\right)\left(1 \otimes c_{X^{*} X, X}^{-1} \otimes 1\right)\left(1 \otimes 1 \otimes b_{X^{*} X}\right)\left({ }^{*} z^{*} \otimes z\right) \\
& =d_{X^{*} X}\left(1 \otimes 1 \otimes d_{X}\right)\left(1 \otimes 1 \otimes c_{X, X^{*} X^{*}}\right)\left(1 \otimes c_{X^{*} X, X}^{-1} \otimes 1\right) \\
& \left(1 \otimes 1 \otimes b_{X^{*} X}\right)\left({ }^{X^{*}} z^{*} \otimes z\right) \\
& =d_{X^{*} X}\left(1 \otimes 1 \otimes d_{X}\right)\left(1 \otimes c_{X^{*} X, X^{*}}^{-1} \otimes 1\right)\left(1 \otimes c_{X^{*} X, X^{*}} \otimes 1\right) \\
& \left(1 \otimes 1 \otimes c_{X, X^{*} X^{*}}\right)\left(1 \otimes c_{X^{*} X, X}^{-1} \otimes 1\right)\left(1 \otimes 1 \otimes b_{X^{*} X}\right)\left({ }^{*} z^{*} \otimes z\right) \\
& =d_{X^{*} X}\left(1 \otimes d_{X_{X}} \otimes 1\right)\left(1 \otimes 1 \otimes c_{X^{*} X, X}\right)\left(1 \otimes c_{X^{*} X, X^{*}} \otimes 1\right) \\
& \left(1 \otimes 1 \otimes c_{X, X^{*} X^{*}}\right)\left(1 \otimes c_{X^{*} X, X}^{-1} \otimes 1\right)\left(1 \otimes 1 \otimes b_{X^{*} X}\right)\left({ }^{X^{*}} z^{*} \otimes z\right) \\
& =d_{X^{*} X}\left(1 \otimes d_{X_{X}} \otimes 1\right)\left(1 \otimes c_{X} X_{X^{*}} \otimes 1\right)\left(1 \otimes 1 \otimes c_{X^{*} X X^{*} X^{*}}\right) \\
& \left(1 \otimes c_{X^{*} X, X} \otimes 1\right)\left(1 \otimes c_{X^{*} X, X}^{-1} \otimes 1\right)\left(1 \otimes 1 \otimes b_{X^{*} X}\right)\left({ }^{*} z^{*} \otimes z\right) \\
& =d_{X^{*} X}\left(1 \otimes d_{X_{X}} \otimes 1\right)\left(1 \otimes c_{X} X_{X^{*}} \otimes 1\right)\left(1 \otimes 1 \otimes c_{X^{*} X, X^{*} X^{*}}\right) \\
& \left(1 \otimes 1 \otimes b_{X^{*} X}\right)\left({ }^{X^{*}} z^{*} \otimes z\right)
\end{aligned}
$$




$$
\begin{aligned}
= & d_{X^{*} X}\left(1 \otimes d_{X_{X}} \otimes 1\right)\left(1 \otimes 1 \otimes c_{X_{X}, X^{*} X}^{-1}\right)\left(1 \otimes c_{X, X^{*}} \otimes 1\right) \\
& \left(1 \otimes b_{X} \otimes 1\right)\left({ }^{X^{*}} z^{*} \otimes z\right) \\
= & d_{X^{*} X_{X}}\left(1 \otimes{ }^{X^{*}} z^{-1}\right)\left({ }^{X^{*}} z^{*} \otimes 1\right) \\
= & d_{X^{*} X}\left(1 \otimes{ }^{X^{*}} z^{-1}\right)\left(d_{X} \otimes 1 \otimes 1\right)\left(1 \otimes{ }^{*} z \otimes 1 \otimes 1\right)\left(1 \otimes b_{\left.X^{*} X_{X} \otimes 1\right)}\right. \\
= & d_{X^{*} X}\left(d_{X} \otimes 1 \otimes 1\right)\left(1 \otimes{ }^{*} z \otimes 1 \otimes 1\right)\left(1 \otimes b_{X^{*} X} \otimes 1\right)\left(1 \otimes{ }^{*} z^{-1}\right) \\
= & d_{X}\left(1 \otimes 1 \otimes d_{X^{*} X}\right)\left(1 \otimes{ }^{X^{*}} z \otimes 1 \otimes 1\right)\left(1 \otimes b_{X^{*} X} \otimes 1\right)\left(1 \otimes{ }^{X^{*}} z^{-1}\right) \\
= & d_{X}\left(1 \otimes{ }^{X^{*}} z\right)\left(1 \otimes 1 \otimes d_{X^{*} X}\right)\left(1 \otimes b_{X^{*} X_{X}} \otimes 1\right)\left(1 \otimes{ }^{*} z^{-1}\right) \\
= & d_{X}\left(1 \otimes{ }^{X^{*}} z\right)\left(1 \otimes{ }^{X^{*}} z^{-1}\right) \\
= & d_{X} .
\end{aligned}
$$

Similarly, one can prove that the arrow $b_{X}: I \rightarrow X \otimes X^{*}$ defines an arrow $(I$, id $) \rightarrow(X, z) \otimes\left(X^{*},{ }^{*} z^{*}\right)$ in $\mathcal{F}^{\prime}$.

Using the lemma above, we obtain a crossed tensor functor $\mathcal{F} \rightarrow \mathcal{F}^{\prime}$ which takes $X$ to $(X, z)$ and takes $X^{*}$ to $\left(X^{*}, X^{*} z^{*}\right)$. Then for any object $U$ in $\mathcal{F}$, the value $\left(U, \theta_{U}\right)$ of this tensor functor at $U$ gives the twist $\theta: U \rightarrow$ ${ }^{U} U$. The uniqueness of the braiding and the twist follows from the same argument in [2]. Let $c, c^{\prime}$ be braidings on $\mathcal{F}$ such that $c_{X, X}=y=c_{X, X}^{\prime}$. For any object $U$ of $\mathcal{F}$, let $\mathcal{E}(U)$ be the set of objects $Z$ for which $c_{U, Z}=c_{U, Z}^{\prime}$, and let $\mathcal{E}$ be the set of objects $U$ for which $\mathcal{E}(U)=\operatorname{obj} \mathcal{F}$. Then using the connectivity structure on $\mathcal{F}$, we see that both $\mathcal{E}(U)$ and $\mathcal{E}$ are closed under tensor product and crossed action. Moreover, using the fact that $u=c_{X, X^{*}}^{-1}$, $v=c_{X^{*}, X}$ and $w=c_{X^{*}, X^{*}}$, we see that $X, X^{*} \in \mathcal{E}(X)$ and $X, X^{*} \in \mathcal{E}\left(X^{*}\right)$. Thus $X, X^{*} \in \mathcal{E}$, and since $X, X^{*}$ generate obj $\mathcal{F}$, we have $\mathcal{E}=\operatorname{obj} \mathcal{F}$. Similarly, one can prove the uniqueness of twist. This completes our proof of Theorem.

\section{References}

[1] Freyd P. and Yetter D., Braided compact closed categories with applications to low dimensional topology. Adv. in Math. 77 (1989), 156-182.

[2] Joyal A. and Street R., Tortile Yang-Baxter operators in tensor categories. J. Pure Appl. Algebra. 71 (1991), 43-51.

[ 3 ] Joyal A. and Street R., The geometry of tensor calculus I. Adv. in Math. 88 (1991), $55-112$.

[4] Kassel C., Quantum groups. GTM. 155, Springer-Verlag, New York 1995.

[5] Kassel C. and Turaev V., Double construction for monoidal categories. Acta. Math. 175 (1995), 1-48. 
[6] Majid S., Representations, duals and quantum doubles of monoidal categories. Rend. Circ. Mat. Palermo (2) Suppl. (1991), 197-206.

[ 7 ] Shum M.C., Tortile tensor categories. J. Pure. Appl. Algebra. 93 (1994), 57-110.

[8] Turaev V., The Yang-Baxter equation and invariants of links. Invent. Math. 92 (1988), 527-553.

[9] Turaev V., Homotopy field theory in dimension 3 and crossed group-categories. GT/0005291.

[10] Yetter D., Markov Algebras, in Braids (J.S. Birman and A. Libgober eds.) AMS Cotemp. Math. Vol. 78 (1988), 705-730.

10-14 Yanagida, Kashiwagimachi Hiraka-machi, Minamitsugaru-gun Aomori 036-0104, Japan

E-mail:colour@mrh.biglobe.ne.jp 